\title{
EL NUDGE DESDE LA PERSPECTIVA DE LA IGUALDAD COMO NO SUBORDINACIÓN
}

\author{
NUDGING FROM AN EQUALITY AS ANTI- \\ SUBORDINATION STANDPOINT
}

\begin{abstract}
Viviana Ponce de León Solís
RESUMEN: En las últimas décadas, el nudge (regulaciones no coercitivas inspiradas en los aportes de las ciencias del comportamiento) ha recibido creciente aplicación, tanto a nivel nacional como comparado, en diversos ámbitos de interés general. Sin embargo, algunos estudios sugieren que esta técnica podría tener un impacto negativo sobre grupos sociales desaventajados. Estas observaciones contrastan abiertamente con la escasa atención que la literatura del nudge da a consideraciones de igualdad en general y de desventaja grupal en particular. Este trabajo aborda esta desatención desde una perspectiva teórica, analizando en qué medida los presupuestos descriptivos y prescriptivos subyacentes al nudge son compatibles con aquellos que subyacen al enfoque de la igualdad como no subordinación.
\end{abstract}

Palabras clave: nudge, arquitectura de decisiones, igualdad, no subordinación, grupos desaventajados.

ABSTRACT: In the last decades, nudging (non-coercive regulations inspired by insights from behavioral sciences) has received growing use, both at national and comparative level, in diverse areas of general interest. However, some studies suggest that this technique could have a negative impact on disadvantaged social groups. These observations overtly contrast with the scant attention that nudging literature gives to equality issues in general and group disadvantage issues in particular. This paper addresses this inattention from a theoretical perspective, analyzing the extent to which the descriptive and prescriptive assumptions underlying nudging are compatible with those underlying the approach of equality as anti-subordination.

Keywords: nudge, choice architecture, equality, anti-subordination, disadvantaged groups.

\section{INTRODUCCIÓN}

En el ultimo par de décadas, el empleo del nudge-denominación popular que se da a un conjunto regulaciones no coercitivas inspiradas en los aportes de las ciencias del comportamiento- se ha hecho cada vez más frecuente. En efecto, esta clase de regulaciones ha proliferado en distintos países, en ámbitos tan diversos como el ahorro previsional, la disminución del consumo energético y la promoción del reciclaje, solo por mencionar algunos ejemplos. Su creciente popularidad se explica porque, de acuerdo con sus promotores, se trataría de una técnica de regulación capaz de influir de forma estadísticamente predecible

\footnotetext{
Doctora en Derecho. Profesora Auxiliar de la Universidad Austral de Chile. Dirección postal: Escuela de Derecho, Universidad Austral, Los Pinos s/n, Balneario Pelluco, Puerto Montt. Correo electrónico: viviana.poncedeleon@uach.cl. Código Orcid: https://orcid.org/0000-0001-7574-1717. Este trabajo forma parte del proyecto FONDECYT Iniciación No 11190199 , en el cual la autora es investigadora responsable.
} 
en el comportamiento de sus destinatarios, a bajo costo y con una afectación mínima de la libertad individual.

Pese a lo anterior, algunas investigaciones teóricas y empíricas sugieren que esta técnica podría tener un impacto negativo sobre grupos sociales desaventajados. Según esas investigaciones, ciertos factores socioeconómicos o barreras estructurales podrían impedir sistemáticamente a los individuos pertenecientes a determinados colectivos sociales adherir (o no) libremente a ella. Esta situación, a su vez, podría redundar en un goce diferenciado de los beneficios derivados de esta técnica y, potencialmente, en un agravamiento de las brechas ya existentes entre los distintos grupos en que se estructura la sociedad.

Estas observaciones contrastan marcadamente con la escasa atención que la literatura del nudge da a consideraciones de igualdad en general y de desventaja grupal en particular. Así las cosas, parece conveniente que dicha literatura subsane esa desatención y entable un diálogo con los estudios sobre igualdad. Este trabajo pretende formular una primera contribución para propiciar ese diálogo, mediante un análisis de la compatibilidad entre la teorización subyacente a la figura del nudge y el marco teórico en que se apoya la concepción de igualdad como no subordinación. A partir de este análisis, se busca precisar si aquella admite consideraciones relativas a la desigualdad estructural de sus destinatarios, que luego se puedan proyectar en el diseño de nudges, o si, por el contrario, excluye categóricamente tal posibilidad.

Al efecto, el trabajo se divide en cuatro partes. En la primera parte se identifican las premisas centrales que justifican el uso de técnicas de regulación inspiradas en los aportes de las ciencias del comportamiento. Luego, en la segunda parte se expone que la igualdad ha tenido una posición muy marginal en la construcción doctrinaria del nudge, a diferencia de otros derechos, principios y valores constitucionales. Esta exposición permite establecer una conexión con la literatura que llama la atención sobre los potenciales efectos redistributivos negativos que podría tener esta figura. A continuación, en la tercera parte se analizan los presupuestos teóricos en que se sustenta el enfoque de la igualdad como no subordinación, bajo el entendido de que constituye la teorización más idónea para canalizar las inquietudes de los críticos al nudge. Finalmente, en la cuarta parte se comparan las dos teorizaciones, destacando sus principales puntos de convergencia y divergencia.

La hipótesis que se pretende demostrar es que algunos de los postulados descriptivos sobre los cuales reposa el nudge no solo son ciegos a consideraciones de desigualdad estructural, sino que pueden incluso estimarse contrarios a ellas. No obstante, existe una consideración clave común al empleo de regulaciones inspiradas en las ciencias del comportamiento y al enfoque de la igualdad como no subordinación, que podría posibilitar su articulación. Esa articulación, en cualquier caso, supondría introducir ajustes o modificaciones a las concepciones dominantes en la actualidad en torno al nudge.

\section{APROXIMACIÓN TEÓRICO-CONCEPTUAL AL NUDGE}

Cualquier aproximación teórico-conceptual al nudge tiene como referencia ineludible el texto de R. Thaler y C. Sunstein, Nudge: Improving Decisions about Health, Wealth, and Happiness (2008), así como algunos de los trabajos posteriores del último en la mate- 
ria. De acuerdo con la primera de estas obras, un nudge "es cualquier aspecto de la arquitectura de las decisiones que modifica la conducta de las personas de una manera predecible sin prohibir ninguna opción ni cambiar de forma significativa sus incentivos económicos" La arquitectura de decisiones, a su vez, alude al modo en que las decisiones son afectadas por el contexto, manera y secuencia en que son presentadas las opciones a elegir, así como por el rango de opciones disponibles.

La construcción doctrinaria del nudge se nutre sobre todo de las contribuciones de las ciencias del comportamiento, como la economía del comportamiento, la psicología cognitiva y la neurociencia social, entre otras ${ }^{2}$. Particularmente significativa entre tales contribuciones resulta la idea central de que la racionalidad humana no es plena ni perfecta, como lo afirmaba el enfoque del análisis económico del derecho. Al contrario, ella se ve afectada por diversos errores o sesgos cognitivos, que pueden influir decisivamente en nuestras decisiones. La promesa de esta figura, entonces, es diseñar regulaciones que tomen en cuenta esos errores o sesgos, a fin de incrementar su eficacia ${ }^{3}$.

\section{TEORÍA DEL PROCESO DUAL}

El punto de partida para la construcción doctrinaria del nudge se encuentra en los aportes de la psicología cognitiva y, más específicamente, en la denominada teoría del proceso dual. Bajo esta denominación se comprende una serie de postulados de carácter descriptivo, y a veces también de carácter prescriptivo, en torno al razonamiento humano ${ }^{4}$. La idea fundamental de esta teoría, según la tradición iniciada por D. Kahneman en su obra Thinking, Fast and Slow (2011), se puede resumir en la distinción entre dos mecanismos modulares de operaciones cognitivas: el Sistema 1 y el Sistema 2.

Bajo el dominio del Sistema 1 se encuentran diversas tareas cognitivas rutinarias que requieren una respuesta veloz, como comprender una expresión en nuestro idioma nativo o dirigir nuestra atención a un sonido fuerte e inesperado 5 . Es por eso que se caracterice al Sistema 1 como rápido, automático e inconsciente ${ }^{6}$.

Para lograr su cometido y evitar la sobrecarga cognitiva, el Sistema 1 se apoya en una serie de intuiciones, heurísticas y sesgos ${ }^{7}$.

Las intuiciones pueden definirse como el reconocimiento de una experiencia previa, directa o indirecta, ante una determinada situación presente. Dicho en otras palabras, la intuición opera cuando una situación nos ofrece una señal que nos conduce (inconscientemente) a información almacenada en nuestra memoria, y esa información nos da la respuesta de cómo actuar en tal situación ${ }^{8}$.

\footnotetext{
1 Thaler y Sunstein (2008) p. 5.

2 Jolls, Sunstein y Thaler (1998) p. 1473.

3 Jolls, Sunstein y Thaler (1998) pp. 1473-1476.

4 Kahneman (2011) pp. 236-237.

5 Kahneman (2011) p. 22.

${ }^{6}$ Kahneman (2011) p. 20

7 TVersky y Kahneman (1974).

8 Kahneman (2011) p. 20.
} 
Las heurísticas, a su turno, pueden ser descritas como "atajos mentales" basados en la experiencia práctica. Estos atajos permiten reducir la complejidad de operaciones de evaluación probabilística de la ocurrencia de un evento incierto, simplificándolas y automatizándolas9. Con todo, dado que la experiencia en que se basa la heurística posee una validez limitada, puede conducir a errores de juicio. Así, por ejemplo, la heurística de representatividad consiste en estimar la probabilidad de que un objeto A pertenezca a una categoría $\mathrm{B}$, considerando la medida en que $\mathrm{A}$ es similar o representativo del estereotipo de $\mathrm{B}^{10}$. Por su parte, la heurística de disponibilidad supone juzgar la probabilidad de ocurrencia de un evento en atención a la facilidad con que un ejemplo o caso se viene a la mente -a partir de la experiencia personal reciente o la mera contingencia, por ejemplo-, pero ignorando otros ejemplos o casos relevantes ${ }^{11}$. Finalmente, la heurística de anclaje y ajuste implica asumir un valor o una información como punto de referencia inicial (anclaje), al cual se van adaptando las creencias y juicios posteriores (ajuste) ${ }^{12}$.

Los sesgos, por último, constituyen desviaciones predecibles, sistemáticas y, en ocasiones, inconscientes en la interpretación de algún aspecto de la realidad objetiva ${ }^{13}$. Ellos resultan de los procesos de categorización y búsqueda de coherencia que subyacen a la cognición humana en general. Su propósito es simplificar la tarea de captar y retener información sobre la realidad circundante y evitar que nuestra capacidad de procesamiento cognitivo se vea abrumada por una cantidad inmanejable de datos. Así, es posible deducir rasgos o propiedades que no son fácilmente observables, inferir relaciones de causalidad entre distintos eventos y, en definitiva, adoptar un determinado curso de acción ante un problema en concreto, incluso antes de tener información completa y precisa a su respecto.

Aunque la literatura especializada ofrece listados prácticamente infinitos de sesgos, tres ejemplos -que corresponden a los sesgos que figuran de manera más prominente en los textos sobre nudge- pueden ser pertinentes para ilustrar su operación. En primer lugar, el sesgo presente implica sobrevalorar una gratificación inmediata a expensas del logro de una meta a largo plazo o la consecución de un bien futuro, aun si el beneficio que conllevan los segundos supera el beneficio de la primera ${ }^{14}$. En segundo lugar, el sesgo statu quo consiste en la tendencia a preferir el estado actual de cosas, por sobre su variación ${ }^{15}$. En tercer lugar, el sesgo optimista se traduce en la inclinación a sobrestimar la probabilidad de eventos positivos y a subestimar eventos negativos futuros ${ }^{16}$.

Como se puede apreciar, las intuiciones, heurísticas y sesgos propios del Sistema 1 actúan como verdaderas estrategias de supervivencia, toda vez que estar "constantemente cuestionando nuestro propio pensamiento sería imposiblemente tedioso" 17 . Mas, pese a su

\footnotetext{
9 TVersky y Kahneman (1974) p. 1124.

10 TVersky y Kahneman (1974) pp. 1124-1127.

11 TVersky y Kahneman (1974) pp. 1127-1128.

12 Tversky y Kahneman (1974) pp. 1128-1130.

13 Haselton, Nettle y Andrews (2015) p. 968.

4 Zamir y Teichman (2014) pp. 88-93.

Zamir y Teichman (2014) pp. 48-50.

16 Zamir y Teichman (2014) pp. 61-64.

17 Kahneman (2011) p. 28
} 
evidente utilidad, ellas no garantizan decisiones óptimas. Y aquí radica la importancia del segundo mecanismo modular de operaciones cognitivas: el Sistema 2. Este último se asocia con tareas que requieren atención y concentración, como operaciones matemáticas y estadísticas o comprender un idioma distinto al nativo. Es así como, en contraste con el Sistema 1, el Sistema 2 se caracteriza como lento, controlado y consciente o, más generalmente, como racional. Esto no significa, en todo caso, que el Sistema 2 sea infalible; es también susceptible a sesgos y puede cometer errores. Sin embargo, es posible reconocer situaciones en las que los errores son más probables y hacer un esfuerzo por disminuir los errores significativos cuando los bienes en juego así lo ameriten ${ }^{18}$.

\section{RACIONALIDAD LIMITADA}

Una de las implicancias normativas más importantes de asumir la teoría del proceso dual como premisa del nudge radica en el concepto de racionalidad limitada (bounded rationality). Este concepto sugiere que la capacidad de las personas para tomar decisiones se encuentra restringida por la cantidad de información que tienen disponible, el tiempo finito con que cuentan y sus propias limitaciones cognitivas, en los términos expuestos en el apartado anterior. He ahí la explicación de porqué algunas personas adoptan ciertos cursos de acción (como gastar en exceso o fumar) aun cuando son inconsistentes con sus metas a largo plazo (ahorrar) o con sus preferencias declaradas (dejar de fumar). También aquí radica la explicación de porqué algunas personas adoptan ciertos cursos de acción aun cuando resultan abiertamente arriesgados y potencialmente peligrosos para otras personas (como conducir a exceso de velocidad).

El origen del término se atribuye a H. A. Simon, autor de la obra Models of Bounded Rationality (1982). En ella, la idea de racionalidad limitada es presentada como una reacción frente a los postulados centrales de la economía neoclásica y, más específicamente, frente a la premisa de racionalidad perfecta que subyace al paradigma del homo economicus. De hecho, es interesante notar que para Simon la noción de racionalidad limitada era una parte importante y adaptativa de la condición humana. En cambio, en la lectura que Thaler y Sunstein hacen de la obra de Simon, la racionalidad limitada de las personas constituye un error, una desviación o una "falla conductual de mercado" (behavioral market failure), que justificaría una intervención estatal correctiva de las decisiones contrarias a las preferencias de quien las adopta o contrarias a los intereses del público en general ${ }^{19}$.

La solución que la figura del nudge ofrece frente a la premisa de racionalidad limitada es el diseño de medidas que obedezcan a una de dos configuraciones. Por un lado, se encuentran las medidas que se apoyan en el Sistema $1-0$, de manera más precisa, en los sesgos, intuiciones y criterios heurísticos distintivos del Sistema 1-, que inducen los procesos automáticos de toma de decisiones en un sentido predeterminado. Son ejemplos de esta primera clase de medidas ${ }^{20}$ :

\footnotetext{
18 Kahneman (2011) p. 28.

19 Sunstein y Thaler (2008) p. 7. También en Sunstein (2014) p. 16.

20 Sunstein (2016a) p. 128-129.
} 
(a) La reglas por omisión, que predeterminan la posición jurídica de una persona, a falta de una manifestación de voluntad en un sentido diverso. Las reglas por omisión explotan el sesgo statu quo, en cuanto se aprovechan de la inercia de las personas y su preferencia por el estado actual de cosas.

(b) La alteración de espacios físicos (o virtuales), que consiste en introducir modificaciones en la configuración de determinados lugares o zonas, para facilitar o dificultar ciertas elecciones.

(c) El encuadre (framing) de información, que significa presentar la información de modo tal que ciertos datos sobresalgan o destaquen por sobre otros. El encuadre de información puede operar como un nudge en la medida que aprovecha la heurística de anclaje y ajuste.

(d) La transmisión de normas sociales, que consiste en presentar información relativa a la conducta que se estima deseable y sobre la cual se espera influir, en términos de lo que otros ya están haciendo.

Por otro lado, se hallan las medidas dirigidas a estimular o activar el Sistema 2, con miras a incentivar la reflexión y la deliberación en la adopción de decisiones. Entre los ejemplos típicos de esta segunda clase de medidas se encuentran ${ }^{21}$ :

(a) La provisión y simplificación de información fáctica, dirigida a promover generalmente la toma de decisiones informadas y, en algunos casos, a contrarrestar el sesgo presente. Este último sería el caso, por ejemplo, del etiquetado nutricional en alimentos envasados.

(b) El uso de advertencias gráficas o textuales, especialmente cuando están destinadas a desactivar el sesgo optimista y resaltar los riesgos que acarrea una determinada actividad, como las advertencias en las cajetillas de cigarros.

(c) Las estrategias de compromiso previo (precommitment strategies), en virtud de las cuales una persona se compromete consigo misma a seguir un determinado curso de acción.

(d) Los recordatorios.

Gracias a estas dos categorías de estrategias, a juicio de los promotores del nudge, este se erigiría en un paradigma regulatorio superior a los que le han precedido. Una de sus ventajas consistiría en ser más eficiente que otras técnicas de regulación (particularmente aquellas basadas en la coerción o en incentivos económicos), ya que conduciría a resultados estadísticamente predecibles en la conducta de sus destinatarios, a un bajo costo. Por otro lado, tendría también la ventaja de ser más eficaz que otras técnicas regulatorias, en la medida que lograría alinear los intereses a largo plazo de sus destinatarios con la consecución de fines de interés general ${ }^{22}$. Por último, ya que no privaría coactivamente a las personas de la posibilidad de escoger alternativas menos óptimas de conducta ni elevaría los costos de escoger en uno u otro sentido, presentaría la ventaja de preservar de mejor manera la libertad individual.

\section{ARQuitectura DE DECISIONES}

El empleo del nudge como técnica regulatoria se adscribiría a la corriente filosófica denominada paternalismo libertario, por Thaler y Sunstein. Dicha corriente alude a "un

\footnotetext{
21 Sunstein (2016a) pp. 127-128.

22 Thaler y Sunstein (2008) p. 7.
} 
tipo de paternalismo relativamente débil y blando, y que no supone ninguna intromisión porque las opciones no se bloquean ni se eliminan, ni se gravan de forma significativa” ${ }^{23}$. En lugar de ello, el paternalismo libertario propone utilizar como táctica la arquitectura de decisiones, que consiste en seleccionar, organizar y presentar las opciones, con el propósito de facilitar la adopción de ciertas decisiones y disuadir otras.

El componente libertario de esta estrategia "radica en la convicción de que, en general, las personas deben ser libres para hacer lo que desean" ${ }^{24}$. Esa libertad, en principio, es concebida en términos acotados, como la mera ausencia de restricciones formales que reduzcan el rango de opciones que pueden ser seleccionadas por un individuo o que hagan su selección más costosa en términos de tiempo, esfuerzo o en cualquier otro sentido ${ }^{25}$. Las personas, entonces, serían "libres para hacer lo que quieran" en la medida que no haya opciones prohibidas ni sanciones o premios significativos asociados a la elección de una o más opciones en particular.

El componente paternalista, por otro lado, estriba en afirmar que es legítimo que los arquitectos de las decisiones influyan en las decisiones de las personas para protegerlas de sus propios errores y sesgos cognitivos. Empero, la protección que se puede proporcionar mediante esta técnica es calificada, pues aspira a "que quienes deciden salgan beneficiados, a juicio de ellos mismos" 26 . El sentido de esta última expresión es enfatizar que se trata de una forma especial de paternalismo en la que el juicio acerca de lo que cuenta como una protección para sus beneficiarios es determinado por ellos mismos y no por el Estado u otro agente.

Ahora bien, establecido que es posible influir en las decisiones de las personas, quedaría por determinar cómo se justifica tal práctica y, muy especialmente, cómo se justifica que el Estado se involucre en tal práctica. Para abordar la cuestión, Thaler y Sunstein apelan especialmente a los potenciales escépticos al componente paternalista de su propuesta, exponiendo que tras la posición escéptica se esconde un supuesto falso y dos equivocaciones.

El supuesto falso es que "casi todas las personas, casi todo el tiempo, eligen lo que es mejor para ellas o, al menos, mejor que si eligieran otras en su lugar" ${ }^{27}$. La falsedad de este supuesto es graficada aludiendo a la dieta y al consumo de tabaco y de alcohol. Respecto de estos dominios, los autores sostienen que "no puede pretenderse razonablemente que las opciones actuales de la gente sean la mejor forma de promover su bienestar" ${ }^{28}$. En esos dominios sería legítimo usar el nudge, para ayudar a las personas a tomar mejores decisiones, según sus propias preferencias. Sería igualmente falso, por extensión, suponer que las opciones actuales de la gente sean la mejor forma de promover el interés general. Así lo evidenciaría la conducta de la mayoría de las personas en ámbitos como el consumo ener-

\footnotetext{
23 Thaler y Sunstein (2008) p. 5.

24 Thaler y Sunstein (2008) p. 5.

25 Nótese que esta concepción de la libertad difiere de aquella que la entiende como el control que un individuo tiene sobre su propia evaluación de las opciones disponibles, la deliberación y la elección, como lo señalan Hausman y Welch (2010) p. 124.

26 Thaler y Sunstein (2008) p. 5.

27 Thaler y Sunstein (2008) p. 9.

28 Thaler y Sunstein (2008) p. 7.
} 
gético doméstico, el reciclaje o la donación de órganos. Por tanto, también sería legítimo emplear el nudge para proteger el medio ambiente o preservar la salud de las personas ${ }^{29}$.

Por lo que refiere a la primera equivocación, esta consistiría creer que es posible evitar influir en las decisiones de las personas. Los autores puntualizan que "ninguna elección carece de contexto", de modo que "no tiene sentido pedir al Gobierno que se mantenga al margen" ${ }^{30}$. En términos más generales, ellos concluyen que "la posición antinudge es improductiva, [porque] literalmente no lleva a ningún sitio" ${ }^{31}$. En obras posteriores, Sunstein ha profundizado en este punto, reiterando que es infructuoso discutir si emplear el nudge resulta ético o no. En lugar de ello, a su juicio, la pregunta que debería plantearse es cuándo resulta ético emplearlo. Y, para responder a este último interrogante, él propone cuatro valores que podrían emplearse como criterios de evaluación: el bienestar, la autonomía, la dignidad y el autogobierno ${ }^{32}$.

Finalmente, en cuanto a la segunda equivocación, esta radicaría en asumir que el paternalismo siempre implica coerción. Thaler y Sunstein subrayan que existen múltiples ejemplos de arquitectura de decisiones que demostrarían que el nudge no fuerza ni impone decisión alguna a sus destinatarios ${ }^{33}$. Siendo así, concluyen: "algunos tipos de paternalismo deberían ser aceptables incluso para quienes más valoran la libertad de elección"34 .

\section{NUDGE E IGUALDAD}

Mediante el recurso al nudge, el Estado puede definir una serie de cualidades y prácticas socialmente deseables, en diversos órdenes de la vida en comunidad. Por ejemplo, en el ámbito del desarrollo de virtudes cívicas destacan las intervenciones dirigidas a impulsar la caridad, a promover el voluntariado, fomentar prácticas inclusivas y concitar el debate, la deliberación y la participación democrática ${ }^{35}$. Respecto de los hábitos de consumo de las personas, se encuentran aquellos que buscan incentivar el reciclaje y compostaje, inducir al ahorro e incitar la austeridad en el consumo de energía, reducir la huella de carbono individual $^{36}$, estimular la ingesta de alimentos saludables, propiciar la actividad física y desincentivar el consumo de alcohol, tabaco u otras sustancias adictivas ${ }^{37}$. Incluso, pueden tener por objeto disuadir determinadas decisiones reproductivas y alentar otras ${ }^{38}$.

En este contexto, no obstante el avivado interés y entusiasmo que suscita el uso del nudge como técnica alternativa de regulación de conductas sociales en ciertos círculos aca-

\footnotetext{
29 Sunstein (2015) p. 427.

30 Thaler y Sunstein (2008) p. 237.

31 Thaler y Sunstein (2008) p. 11.

32 Sunstein (2016b) pp. 105-145.

33 Thaler y Sunstein (2008) p. 11.

34 Thaler y Sunstein (2008) p. 11. Sin perjuicio de lo anterior, se ha clarificado que no todo nudge es por definición paternalista más recientemente en SunSTEIN (2015) p. 433.

35 Button (2018) pp. 1034-1052; John y otros (2011) pp. 43-54.

36 John, SMith y STOKer (2009) pp. 361-370.

37 Quigley (2013) pp. 588-621.

38 Persad (2014) pp. 273-298.
} 
démicos y políticos, existen severas críticas a esta figura. Las críticas surgen debido a que la definición de los fines de interés colectivo cuya satisfacción se persigue con la creación de nudges puede conllevar una definición estatal del "bienestar individual" e incidir de manera significativa en las opciones de vida de las personas. Ello, a su vez, podría significar una afectación ilegítima de diversos derechos, principios y bienes constitucionales.

\section{El ROL DE LA IGUALDAD EN El DESARROlLO TeÓRICO DEL NUDGE}

Desde una perspectiva constitucional, los cuestionamientos al nudge suelen apuntar al menos a tres aspectos. En primer lugar, se teme un menosprecio por la dignidad humana, en especial tratándose de aquellos nudges que se aprovechan de los sesgos propios del Sistema $1^{39}$. En segundo lugar, se advierte un riesgo de afectación de la libertad individual, en cuanto a su proyección en el control sobre la valoración de las opciones disponibles al tomar una decisión ${ }^{40}$. Y, en tercer lugar, se repara en una amenaza de invisibilización del actuar estatal, que podría determinar su sustracción del control democrático y jurisdiccional, sobre todo cuando consisten en intervenciones en espacios físicos o virtuales que no son fácilmente perceptibles ${ }^{41}$.

Llama la atención que en el desarrollo de estos cuestionamientos se ha prestado escasa atención a la garantía de igualdad. Algunas publicaciones han puesto de relieve esta omisión, formulando una crítica generalizada a la misma, particularmente desde la perspectiva de la filosofía jurídica ${ }^{42}$. Otras, de carácter empírico, han puesto de relieve el potencial impacto redistributivo desfavorable que ciertas modalidades específicas de nudge podrían tener sobre determinados grupos sociales ${ }^{43}$. Así puede verse, por ejemplo, respecto de la provisión de información y del uso de advertencias gráficas o textuales en alimentos envasados $^{44}$ o en relación con el uso de advertencias gráficas o textuales en los empaques de las cajetillas de cigarros ${ }^{45}$.

La omisión apuntada no deja de ser interesante si se considera que la igualdad se encuentra íntimamente vinculada a otros valores y principios que tienen un rol preponderante en la construcción teórica del nudge, como la autonomía y la libertad individual. Más interesante todavía es observar que el propio Sunstein admite en varias ocasiones, aunque sin profundizar en absoluto en el punto, que ciertos factores estructurales de desigualdad pueden incidir en la eficacia del nudge $e^{46}$. Pero, pese a ello, al identificar y desarrollar los

\footnotetext{
39 Alemanno y Spina (2014) p. 446; Brownsword (2014) pp. 355-358; Cassesse (2016) p. 245; Goodwin, (2016) p. 285; Sunstein (2015) p. 430; Van Aaken (2015) p. 123; Yeung (2011) pp. 1-29.

40 Baldwin (2014) pp. 836-837; Bovens (2009) p. 208; Hansen (2013) pp. 3-28; Hausman y Welch (2010) p. 128; John y STOKer (2017) p. 12; MCCRUDden y King (2016) pp. 104-111; WALdron (2014); WiLKINSON (2012) pp. 341-355; YeUnG (2012) p. 136.

41 Alemanno y Spina (2014) p. 432; Baldwin (2014) p. 845; Cassese (2016); Van Aaken (2015) p. 108.

42 Roberts (2018) pp. 1045-1066; Wells (2010) pp. 115-116.

43 Por ejemplo, en este sentido, National Advisory Committee on Bioethics (2015) p. 15.

44 Araya y otros (2018); Drichoutis, Lazaridis y Nayga (2005); Kim, Nayga y Capps (2001).

45 Cantrell y otros (2013) pp. 1-11; Thomas y otros (2008) pp. 230-237.

46 Sunstein (2016b) pp. 174-175, 179, 193-194.
} 
principios y valores que a su entender deberían guiar el diseño e implementación del nudge -bienestar, autonomía, dignidad y autogobierno-, la igualdad queda excluida ${ }^{47}$.

\section{EL IMPACTO DEL NUDGE SOBRE GRUPOS DESAVENTAJADOS}

Existe una explicación relativamente plausible para la desatención a la garantía de igualdad en la literatura sobre el nudge. Dicha literatura asume como punto de partida un presupuesto igualitario implícito: que todas las personas conducen por igual sus procesos de toma de decisiones, conforme a las operaciones propias de los dos sistemas de pensamiento y que, por lo tanto, son igualmente susceptibles a la influencia del nudge. Por ende, siguiendo todavía a Thaler y Sunstein en este punto, todas las personas serían igualmente capaces de escapar o adherir libremente a él, según sus preferencias personales ${ }^{48}$. En vistas a este presupuesto igualitario, podría pensarse equívocamente que esta figura está a salvo de reparos en clave de igualdad constitucional. Sin embargo, diversas circunstancias pueden incidir de modo determinante en la manera en que distintos individuos responden a esta clase de medidas.

Una de las ventajas teóricas que supone el nudge, en comparación con otras técnicas alternativas de regulación, es que sus destinatarios cuentan con la posibilidad de escapar a él y sustraerse de sus efectos, si es que estos no se ajustan a sus genuinas preferencias. Mas, esta caracterización del nudge no considera suficientemente que pueden existir barreras y obstáculos materiales que impidan a determinados individuos esquivarlo. Así, por ejemplo, la exigencia de renunciar por escrito a una regla por omisión podría representar un obstáculo considerable para personas analfabetas, pertenecientes a minorías lingüísticas o con cierto tipo de discapacidades sensoriales ${ }^{49}$. Otros factores estructurales de desigualdad pueden tener un efecto similar. En este sentido, existe una "creciente evidencia de que aquellos que son menos educados, y menos sofisticados, son más propensos a adherir a una regla por omisión", aun cuando les resulte perjudicial ${ }^{50}$.

Del mismo modo, aunque la decisión hacia la cual se orienta la medida sea ajustada a las preferencias de sus destinatarios, pueden existir barreras u obstáculos materiales que les impidan adherir a la misma ${ }^{51}$. Esta hipótesis puede ser graficada por el comportamiento de ciertos segmentos de consumidores frente al sistema de etiquetado nutricional y a los sellos gráficos de advertencia "alto en", en los alimentos envasados. En este sentido, algunas investigaciones sugieren que "los individuos de mayores ingresos, más educados, tienden a responder en mayor medida a la presencia de las etiquetas en la parte frontal de los envases”, en comparación con los individuos de menores ingresos ${ }^{52}$. Otro tanto ocurre con ciertos tipos de recordatorios que, según lo evidencian algunos estudios en el ámbito del pago

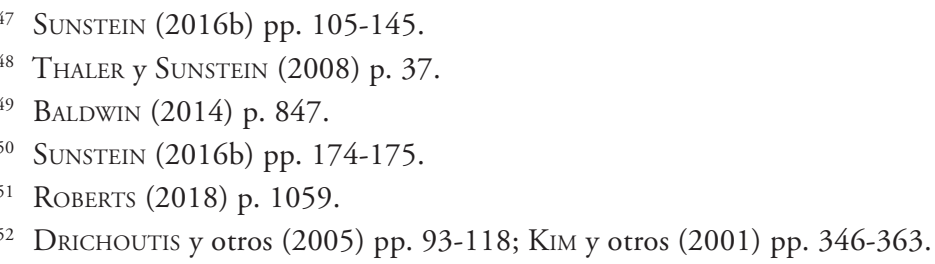


de multas por estacionar en lugares prohibidos, son desproporcionadamente menos eficaces tratándose de grupos desaventajados ${ }^{53}$.

Todo lo anterior, a su vez, podría redundar en un desigual goce de los beneficios sociales que se persiguen mediante la adopción de esta clase de medidas. Esta desigualdad desde luego resulta problemática en cuanto a su impacto inmediato sobre grupos desaventajados que, por lo demás, suelen ser el grupo social en que se presenta con más prevalencia el hábito o conducta que se pretende modificar con el nudge. No obstante, resulta aún más problemático considerar el impacto a largo plazo de esa desigualdad ${ }^{54}$. Considérese, una vez más, el caso del etiquetado nutricional y de los sellos en alimentos envasados. Uno de los principales grupos objetivos de la medida serían las personas de menores ingresos, pues entre ellas se concentrarían las tasas más altas de obesidad ${ }^{55}$. Con todo, si es efectivo que las personas de menores ingresos responden de peor manera al etiquetado y a los sellos, los beneficios sociales de la medida terminarán concentrándose entre las personas de mayores ingresos, con lo cual se ampliará progresivamente la brecha de salud entre unas y otras. Más generalmente, en el ámbito de la salud, cierta evidencia sugiere que las intervenciones estatales centradas en el cambio de comportamiento no son aptas para reducir las desigualdades sociales y que, incluso, podrían agravarlas ${ }^{56}$.

Estas observaciones conducen ineludiblemente a la necesidad de hacer una relectura del nudge. Ellas ponen de manifiesto que la eficacia de estas medidas no depende tan solo de su aptitud para apelar a -o corregir- las intuiciones, sesgos y heurísticas que guían los procesos de adopción de decisiones. Antes bien, tal eficacia se encuentra en alguna extensión condicionada por ciertos factores socioeconómicos y barreras estructurales que son causa de desigualdad social.

\section{IGUALDAD COMO NO SUBORDINACIÓN}

La igualdad es un principio ampliamente aceptado, pero que carece de significado unívoco $^{57}$. En la mayoría de los textos constitucionales contemporáneos se han incorporado cláusulas de igualdad formal y de no discriminación, que reflejan una concepción de la igualdad de corte eminentemente individualista y centradas en la razonabilidad de las diferencias de trato $^{58}$. Pero este aspecto de la igualdad no parece ser el que preocupa a los críticos del nudge. Al contrario, sus inquietudes se centran más en los grupos que en los individuos. En este sentido, la lectura de la igualdad como no subordinación podría resultar más adecuada para canalizarlas.

El origen de esta interpretación del derecho a la igualdad se atribuye especialmente a la obra de Owen Fiss, Groups and the Equal Protection Clause (1976), y sus subsiguientes

\footnotetext{
53 Heffetz, O’Donoghue y Schneider (2020) pp. 1-31.

54 ROBERTS (2018) p. 1061.

55 Araya y otros (2018) pp. 1-43.

56 LORENC y otros (2011) p. 191.

57 Para una caracterización de las distintas interpretaciones que admite la igualdad, véase EsPARZA (2017) pp. 23-44.

58 Fiss (1976) pp. 108
} 
reelaboraciones. Según este autor, el derecho a la igualdad debe ser entendido sobre todo, aunque no exclusivamente, como un mecanismo protección para grupos desaventajados frente a situaciones de discriminación estructural. Para él, el problema central no está en las normas jurídicas en sí mismas sino que en ciertas jerarquías que se perpetúan en la estructura social, las cuales simplemente se proyectan al derecho positivo. La idea central de su tesis es que la garantía constitucional deviene irrealizable bajo condiciones de estratificación social generalizada. De ahí que su propuesta se dirija a desmantelar estructuras sociales injustas, por medio de la impugnación de prácticas estatales o privadas aparentemente neutras y la adopción medidas de acción afirmativa.

\section{GRUPOS SOCIALES}

La concepción de la igualdad como no subordinación se construye a partir de dos presupuestos fundamentales. El primero es que la sociedad se organiza o estructura a partir de distintos grupos sociales. El segundo es que, entre el universo de grupos sociales existentes, algunos de ellos se encuentran en una especial posición de desventaja o vulnerabilidad en comparación con los demás. Resulta así central bajo esta concepción determinar qué rasgos o características permitirían calificar a un grupo social como desaventajado o vulnerable. La cuestión es sin duda controversial y existen múltiples propuestas al respecto ${ }^{59}$. Pese a lo anterior, parece existir cierto consenso entre los adherentes al enfoque de la igualdad como no subordinación en considerar como relevantes al menos tres factores.

El primer factor consiste en que el grupo en cuestión debe calificar como grupo social. Siguiendo a Fiss, los grupos sociales son colectivos de personas, que presentarían ciertas características distintivas que permitirían diferenciarlos de una simple conglomeración accidental o transitoria de personas. Así, en primer lugar, un grupo social constituye una entidad en sí mismo, lo cual significa que tiene una existencia que trasciende a la existencia de sus integrantes en un momento dado y que tiene una identidad que lo hace reconocible $e^{60}$. Luego, en segundo lugar, un grupo social se caracteriza porque existe una condición de interdependencia entre sus miembros, de modo que la identidad y bienestar de estos depende de la identidad y bienestar de aquel, y viceversa ${ }^{61}$.

Es posible definir a los grupos desaventajados a partir de distintos tipos de rasgos. Esos rasgos pueden tener raíces biológicas y ser inmutables (por ejemplo, ciertas discapacidades o la raza), pero no es imprescindible que sea así (por ejemplo, la religión) ${ }^{62}$. Antes bien, lo importante es que se trate de rasgos moralmente irrelevantes para definir la posición de un individuo en sociedad ${ }^{63}$.

El segundo factor consiste en que el grupo debe haber experimentado una historia de discriminación, hostilidad o exclusión, cuyos efectos subsisten y se proyectan a su situación

\footnotetext{
59 Para una discusión, véase Fiss (2004) pp. 1-25.

${ }^{60}$ FISS (1976) p. 148. Para una profundización en la noción de identidad como componente central de un grupo social, véase Young (1990) p. 172.

61 FISS (1976) p. 148.

62 Fiss (1976) p. 155.

${ }^{63}$ FISS (1976) p. 159.
} 
actual. Dicha historia, a su vez, genera dinámicas sociales que producen resultados sistemáticamente desfavorables para los integrantes del grupo y que conducen, en definitiva, a la estratificación de la sociedad. En este contexto, no es posible atribuir la situación de desventaja grupal a una conducta o trato en concreto que pueda calificarse jurídicamente como discriminatorio. El derecho, por su parte, no es necesariamente la causa de esas dinámicas, pero sí un factor que contribuye a reproducirlas y exacerbarlas.

Fiss está consciente de que la orientación retrospectiva de la igualdad como no subordinación conlleva serias dificultades probatorias. Exige que los tribunales reconstruyan nexos causales entre comportamientos agregativos -que incluyen marcos institucionales como prácticas sociales informales- y la minusvaloración de ciertos grupos sociales por períodos extensos de tiempo ${ }^{64}$. Requiere, además, una evaluación de su estatus económico y de su poder político. Ante estas dificultades, Fiss propone el concepto de discriminación de facto como estrategia alternativa no orientada al pasado. Este concepto atiende al impacto desfavorable que ciertas decisiones (por ejemplo, los procesos de selección universitaria o laboral) tienen sistemáticamente sobre determinados grupos.

El tercer y último factor consiste en que el grupo social debe encontrarse en una posición de subordinación persistente. Bajo el enfoque de la igualdad como no subordinación, una vez que un individuo es adscrito socialmente a un determinado grupo, esa adscripción será decisiva en la posición social y económica que ocupará en su comunidad y en el tratamiento que recibirá por parte de sus miembros. Dicha adscripción limitará su nivel de ingreso, sus posibilidades de acceso a bienes básicos (por ejemplo, la educación, la salud, el empleo y la justicia), el ejercicio de sus libertades políticas y, más generalmente, sus proyectos de vida ${ }^{65}$. De acuerdo con Fiss, esas limitaciones proceden de tres fuentes, que pueden operar en forma alternativa o acumulativa: su carencia de poder político, su estatus económico y los prejuicios sociales que existen en su contra ${ }^{66}$.

Sobre esta última cuestión, Fiss destaca que los grupos vulnerables son vistos con "miedo, odio y disgusto" ${ }^{6}$. Efectivamente, la pertenencia a esos grupos suele estimarse indiciaria de ciertas características socialmente indeseables o minusvaloradas (debilidad, proclividad al comportamiento criminal, inutilidad, etc.), cuyo concreto contenido depende del grupo de que se trate. Esta clase de asociaciones, "provoca que les resulte difícil formar coaliciones y alianzas con otros grupos [...] y contribuye a que los demás les inflijan daños o los utilicen como chivo expiatorio" ${ }^{18}$.

\section{JUSTIFICACIONES DE LA IGUALDAD COMO NO SUBORDINACIÓN}

La igualdad como no subordinación supone que ciertas prácticas sociales (que incluyen la discriminación, pero que no están limitadas a ella) deben ser condenadas porque

\footnotetext{
64 Fiss (1976) p. 145.

65 Fiss (1976) p. 162.

66 FISS (1976) p. 152. A falta de un mayor desarrollo del concepto de subordinación por parte del autor, puede resultar esclarecedora la sistematización de sus cinco dimensiones (explotación, marginación, falta de poder, imperialismo cultural y violencia) en Young 1990 pp. 39-65.

67 FISS (1976) p. 152.

${ }^{68}$ Fiss (1976) p. 152.
} 
crean, agudizan o perpetúan las causas estructurales de desventaja que afectan de manera especial a ciertos grupos ${ }^{69}$. En términos generales, el sentido de esta lectura de la igualdad es lograr la igualdad efectiva o material en el goce de los derechos. Bajo este respecto, la erradicación de las estructuras de subordinación puede considerarse como un imperativo de justicia social ${ }^{70}$.

En concreto, Fiss justifica los esfuerzos por mejorar el estatus de los grupos desaventajados en una teoría sobre la compensación. Esta compensación -manifestada en medidas redistributivas o en medidas de acción afirmativa, según se explicará en el siguiente apartado- es debida por la sociedad como un todo al grupo en cuestión, por haber situado a este último en una posición de subordinación ${ }^{71}$. Dicho en otras palabras, la sociedad tendría una responsabilidad colectiva frente a los grupos desaventajados, en cuanto partícipe de una serie de prácticas e instituciones que históricamente les han mantenido en una situación de inferioridad jerárquica ${ }^{72}$. Se trata, entonces, de una justificación que atiende al pasado; a la historia de discriminación sufrida por el grupo.

Sin embargo, este razonamiento no explicaría por qué se debería mejorar el estatus de grupos sociales que, aun siendo blanco de exclusión y prejuicios actuales, no se han encontrado en una posición histórica de subordinación (por ejemplo, migrantes recién llegados). Por ello, Fiss ofrece una segunda justificación que prescinde de la noción de compensación y que, en cambio, se apoya en tres valores comunitarios cuya consecución podría vincularse con la erradicación de la subordinación ${ }^{73}$. Entre tales valores se encuentran:

(a) La preservación de la paz social. Para ilustrar esta idea, Fiss utiliza el ejemplo de la prohibición de la difusión de imágenes y materiales sexualmente explícitos que puedan conducir a la violencia sexual ${ }^{74}$. La razón por la que ciertas jurisdicciones prohíben esta clase de prácticas no es precisamente porque causen un daño directo, sino porque generan o refuerzan ciertas asociaciones mentales que perpetúan la subordinación. En un sentido opuesto, Fiss asocia también la preservación de la paz social con la mantención del orden público. La idea detrás de esta asociación es que ciertos grupos atribuirían una importancia de tal magnitud a su inclusión, que estarían dispuestos a obtenerla incluso por medios violentos $^{75}$. Por ende, si el derecho no posibilita tal inclusión, se corre el riesgo de que aquellos grupos busquen obtenerla por medios ilícitos.

(b) La mantención de la comunidad como un todo cohesivo. Así, por ejemplo, Fiss sostiene que la discriminación basada en el género es reprochable no solo porque implica un tratamiento injusto para ciertas personas, sino porque ese tratamiento injusto subordina al grupo al que pertenecen como un todo, generando jerarquías sociales ${ }^{76}$. Por otro lado, el autor sostiene que la sociedad parece comprometida con la integración en la educación, en

69 FISS (2004) p. 4.

70 Fiss (2004) p. 8.

71 FisS (1976) p. 150.

72 FisS (1971) p. 248.

73 Fiss (1976) p. 151.

74 FISS (1992) pp. 2048 ss.

75 FISS (1971) p. 246.

76 FISS (1994) pp. 417-418. 
el entendido de que las políticas educacionales segregacionistas son indeseables en términos educativos o que constituyen una vulneración de la igualdad ante la ley ${ }^{77}$.

(c) Permitir el más pleno desarrollo de los miembros individuales del grupo. El principio de no subordinación condena las estructuras sociales jerárquicas porque impiden el ideal de autorrealización individual y porque violentan las concepciones de comunidad basadas en ese ideal ${ }^{78}$. Por el contrario, a medida que los miembros de grupos desaventajados ganan acceso a posiciones originalmente reservadas a grupos privilegiados (en educación, empleo, política, etc.), desarrollan autoconfianza y orgullo. Ello, a su vez, podría tener consecuencias sociales beneficiosas ${ }^{79}$. Bajo esta perspectiva, como lo ha explicado Fiss en reiteradas oportunidades el fundamento de la no subordinación sería la igual capacidad de autogobierno de las personas ${ }^{80}$. Por ende, el sentido de la garantía constitucional de la igualdad sería permitir a cada individuo perseguir el más amplio rango de oportunidades para construir y desarrollar el proyecto de vida de su elección. Desde esta perspectiva se subraya que las decisiones individuales están socialmente condicionadas por patrones de discriminación estructural y no son del todo libres.

En línea con lo anterior, se ha propuesto también sustentar la prohibición de la subordinación en el principio democrático. El vínculo entre aquella y este resultaría evidente si se toma en cuenta que una de las proyecciones de la subordinación es la ausencia de poder político. Desde este punto de vista, la marginación estructural de ciertos grupos sociales puede impedir el funcionamiento de la democracia ${ }^{81}$ al desconocer el derecho de sus integrantes a ser tratados como un miembros respetados, responsables y participantes de la sociedad organizada ${ }^{82}$.

\section{ACCIONES AFIRMATIVAS}

A la luz de todo lo anterior, Fiss concluye que la cláusula constitucional de la igualdad debe interpretarse como una prohibición de subordinación. En consecuencia, dicha cláusula no solo prohibiría la acción estatal discriminatoria, sino que tendría un alcance más amplio. Cualquier conducta estatal que genere, agrave o perpetúe la subordinación de grupos vulnerables constituiría una violación de aquella ${ }^{83}$. Más concretamente, esta prohibición de subordinación se traduciría en dos clases de deberes:

En primer lugar, el Estado tendría un deber negativo o de no hacer, que consistiría en no dar un trato arbitrario desfavorable o de no discriminar con base a la sola pertenencia de un individuo a un determinado grupo social ${ }^{84}$. Por ende, el Estado debería abstenerse de excluir directamente a los miembros de un grupo desaventajado del goce y acceso a bie-

\footnotetext{
77 Fiss (1971) p. 247.

78 FISS (1994) p. 421.

79 Fiss (1971) p. 247.

80 Fiss (1992) p. 2044; (1987) p. 785; (1986) p. 1410.

81 Breyer (2005) p. 83.

82 Karst (1977) p. 4.

83 FISS (2004) p. 11.

${ }^{84}$ FISS (1976) p. 157-160.
} 
nes e instituciones públicos ${ }^{85}$. Adicionalmente, el Estado debería abstenerse de promover e incurrir en prácticas que tengan un efecto excluyente en los hechos, aun cuando no involucren una discriminación directa y aun cuando tales prácticas se basen en criterios aparentemente neutrales o inocentes ${ }^{86}$.

En segundo lugar, el Estado tendría un deber positivo o de hacer, que admitiría dos proyecciones. Por un lado, este deber positivo incluiría la prevención y reparación de las vulneraciones a los derechos de los integrantes de grupos desaventajados. De esta manera, la prohibición de la subordinación se extendería también a la acción privada, imponiendo al Estado la obligación de contrarrestarla ${ }^{87}$. Por otro lado, el Estado tendría un deber de adoptar medidas redistributivas o medidas de acción afirmativa, dirigidas a superar la situación de subordinación del grupo.

En la obra de Fiss el énfasis está puesto fundamentalmente en las medida de acción afirmativa. Estas últimas brindan un trato preferencial a los integrantes de los grupos desaventajados, dándoles una mayor participación en las posiciones de poder, prestigio e influencia en la sociedad, que aquella a la que podrían acceder por sí mismos, de no mediar la intervención estatal. Constituyen ejemplos de esta clase de medidas las cuotas electorales, los programas de ayuda económica y el acceso preferencial a la educación superior o a ciertos empleos. Su propósito no es tan solo mejorar la posición de sus beneficiarios concretos, individualmente considerados, sino mejorar la posición relativa del grupo como un todo dentro de la comunidad. Ello ocurriría progresivamente, en la medida que cada vez más miembros del grupo ocupen posiciones que en algún momento fueron ocupadas de forma exclusiva por los integrantes de los grupos sociales más privilegiados ${ }^{88}$.

La lógica que inspira a estas medidas es remediar las injusticias pasadas que han experimentado los grupos desaventajados y protegerlos de las consecuencias de carecer de poder, especialmente político ${ }^{89}$. Al efecto, Fiss asigna un rol particularmente prominente a los tribunales, justamente para rectificar su exclusión del proceso político. Para hacer operativas estas ideas, los tribunales "tendrían que imaginar un universo de posibles medidas que podrían ser adoptadas (por el legislador) a nombre del grupo desaventajado, hacer una elección entre las permisibles, imponer su ejecución y luego controlar a las fuerzas policiales"90. En este sentido, en su opinión, los jueces tendrían un rol crucial en la concreción del ideal de igualdad ${ }^{91}$.

\footnotetext{
85 FISS (1976) p. 160-164.

86 FISS (1976) p. 158.

87 Originalmente esa hipótesis se había descartado en Fiss (1976) pp. 168-170. Sin embargo, con posterioridad el autor rectificó su postura inicial e incorporó a las conductas de los particulares dentro del alcance del principio de igualdad como no subordinación. Véase Fiss (1996a) p. 21; (1996b) p. 11 y (2004) p. 8.

88 Fiss (2004) p. 6.

${ }^{89}$ FISS (1976) p. 153.

90 FISS (1976) p. 170.

91 FISS (1976) p. 176-177.
} 


\section{V. ¿ADMITE EL NUDGE CONSIDERACIONES DE DESVENTAJA GRUPAL?}

En este punto del desarrollo de la presente investigación, es posible discutir en qué medida las premisas teóricas subyacentes al nudge son compatibles con las premisas teóricas subyacentes al enfoque de la igualdad como no subordinación. Esa discusión es relevante para atender adecuadamente a las críticas en torno a los potenciales impactos desfavorables del primero sobre grupos desaventajados. En efecto, si se concluyera que unas y otras son incompatibles, podría suponerse razonablemente que esos impactos desfavorables son inevitables, en cuanto se deben a discrepancias conceptuales y normativas irreconciliables entre ambos constructos, que inciden de manera determinante en el diseño del nudge. A la inversa, si se concluyera que unas y otras son compatibles, esos impactos desfavorables podrían verse como tensiones específicas que pueden ser resueltas o armonizadas en el diseño de estas clase de medidas.

\section{AutONOMÍA, INTERVENCIÓN ESTATAL Y CATEGORÍAS DE DESTINATARIOS}

Una primera diferencia que salta a la vista al comparar la teorización en torno al nudge, según Thaler y Sunstein, con la tesis de la igualdad como no subordinación, según Fiss, es que una y otra toman como punto de partida presupuestos descriptivos radicalmente distintos. La primera concibe a las personas como sujetos neutros en cuanto a sus rasgos individuales y sociales, cuya autonomía se ve restringida por barreras cognitivas (sus propias intuiciones, heurísticas y sesgos), que determinan su racionalidad limitada. En cambio, la segunda concibe a las personas como sujetos cuya autonomía se ve restringida por barreras sociales de carácter estructural, dadas por su adscripción a un determinado grupo social que se encuentra en una posición histórica de subordinación. Como se puede apreciar, la autonomía constituye un valor clave en ambas construcciones, pero con un sentido y objetivo muy distinto en cada caso.

Esta primera diferencia, a su vez, se proyecta en una segunda, ahora en cuanto a los presupuestos prescriptivos y las implicancias normativas que derivan de asumir un enfoque u otro. De acuerdo con la literatura sobre el nudge, la racionalidad limitada de las personas plantea un problema de déficit de autonomía cuya raíz se encuentra en cada individuo; no en el mercado, la sociedad u otras instituciones. La consecuencia práctica que se sigue de ello es que la intervención estatal ha de centrarse, precisamente, en los individuos, dándose por descontada su capacidad material para elegir y decidir. Por el contrario, desde el enfoque de la igualdad como no subordinación, el déficit de autonomía está dado por condiciones estructurales o barreras sociales; factores externos a los individuos. Es así como, en este caso, la intervención estatal está dirigida a erradicar las condiciones sociales que generan subordinación grupal.

En directa relación con lo anterior, la tercera diferencia tiene que ver con la posición que cada teorización adopta frente a la intervención estatal. Para el caso del nudge, esa posición se resume en la filosofía del paternalismo libertario, que aspira a un compromiso entre el bienestar social y la libertad de elección. Dicha filosofía apela especialmente al ethos individualista de la cultura jurídico-política norteamericana y promueve una intervención estatal excepcional y mínima. Por su parte, la tesis de la igualdad como no subordinación 
promueve la intervención estatal enderezada a mejorar el estatus del grupo, sea en la forma de medidas redistributivas que contrarresten su posición de desventaja o en la forma de represión de actos discriminatorios que perpetúen o agraven esa posición. Desde esta perspectiva, la intervención estatal sistemática y regular es vista como una herramienta indispensable para desmantelar estructuras sociales de subordinación.

Para cerrar este punto, existe una cuarta diferencia en torno al modo en que discursivamente se construyen las categorías de destinatarios de cada una de estas intervenciones. Así, los destinatarios del nudge no son definidos en atención a sus características individuales o grupales, sino que son definidos en función de fallas conductuales de mercado. Dicho de otro modo, ellos son definidos en función de aquellas categorías de comportamientos en los cuales las decisiones individuales puedan tener efectos desfavorables para quienes las adoptan o para otros ${ }^{92}$. Por otro lado, la lógica del enfoque de la igualdad como no subordinación es distinta. La intervención estatal se justifica por ciertas características individuales y grupales de sus destinatarios, que han determinado su posición histórica de desventaja social. Desde esta perspectiva es posible distinguir a los destinatarios teóricos del nudge, en cuanto segmentos de la población que constituyen un riesgo para sí y para otros, de la categoría de grupos desaventajados, en cuanto segmentos de la población que se encuentran en una situación de riesgo.

Sobre la base de estas diferencias es posible concluir que hay ciertos elementos en la configuración teórica del nudge que se encuentran en clara contradicción con aquellos en que se sustenta la concepción de la igualdad como no subordinación. Estos elementos impedirían incorporar a la primera, sin más, consideraciones de desigualdad estructural o desventaja grupal. Incluso podría conjeturarse que los impactos negativos del nudge sobre grupos desaventajados que parte de la doctrina denuncia no son la sola consecuencia de una mera inadvertencia u omisión teórica, sino el resultado inevitable de aplicación práctica de sus premisas básicas. Con todo, esta conjetura podría resultar prematura. Queda todavía por examinar, según se explica en el siguiente apartado, otro aspecto de la comparación que podría ser relevante para resolver el asunto.

\section{IMPORTANCIA DEL CONTEXTO}

Un aspecto adicional de la comparación entre la teorización relativa al nudge y la tesis de la igualdad como no subordinación apunta a la importancia que una y otra atribuyen al contexto. En el primer caso, esa importancia se puede apreciar a partir de la propia noción de arquitectura de decisiones, en cuanto se refiere a la manera de organizar el contexto en el que se toman las decisiones ${ }^{93}$. Aquí, el contexto alude al formato (sencillez, tamaño, ubicación espacial, etc.), la secuencia (orden o preferencia) o el énfasis (mayor o menor prominencia) con que se presentan las opciones. En el segundo caso, en cambio, el contexto tiene relación con la historia de determinados grupos sociales y con las distintas barreras que enfrentan, en términos del acceso a bienes básicos y del ejercicio de sus derechos

\footnotetext{
92 Gandy y Nemorin (2018) p. 2119.

93 Véase arriba la nota 1 y el texto a que se refiere.
} 
fundamentales. Este no es fijo para quienes integran el grupo en cuestión, sino que puede variar según su pertenencia simultánea a otros grupos u otros factores.

La irrelevancia del contexto personal de quien toma las decisiones (su sexo, raza, edad, etc.) en la teorización relativa al nudge no es accidental. Es el resultado esperable de asumir una preferencia explícita por la economía del comportamiento, entre todas las posibles disciplinas que integran las ciencias del comportamiento, como principal enfoque disciplinar para desarrollar aquella teorización. Efectivamente, tal como lo ha destacado alguna doctrina, los postulados económicos en general tienden a servir de base para la construcción de modelos abstractos de comportamiento, que son indiferentes a las particularidades de cada individuo ${ }^{94}$. En este orden de ideas, conviene recordar que la versión del nudge popularizada por Thaler y Sunstein no pretendía apartarse radicalmente de los postulados básicos de la economía clásica y del análisis económico del derecho -entre los que destacan el individualismo metodológico y la figura del homo economicus-, sino mejorarlos y hacerlos más realistas ${ }^{95}$. No es de extrañar, entonces, que esta específica versión del nudge tenga un fuerte foco en los individuos (o, más bien, en un modelo abstracto sobre cómo se comportan los individuos) y no en los grupos. Es más, el énfasis de Thaler y Sunstein en la calificación de los sesgos y heurísticas como errores o desviaciones que deben predecirse y corregirse ha sido interpretado como un intento por preservar, antes que superar, la centralidad de los referidos postulados ${ }^{96}$.

Con todo, esta opción metodológica tiene un costo no menor, en cuanto puede conducir a una excesiva simplificación de los fenómenos psicológicos ${ }^{97}$. Así las cosas, la economía del comportamiento solo ofrece una visión parcial de la complejidad del comportamiento, emoción, percepción, cognición, intención y acción humanas, con particular énfasis en la idea de racionalidad y en la respuesta de las personas a incentivos económicos en términos amplios ${ }^{98}$. De ahí su desatención a la manera en que factores culturales, sociales y políticos influyen en el comportamiento de las personas ${ }^{99}$.

Pese a lo dicho, la importancia que las dos construcciones objeto de la presente investigación asignan al contexto es el punto que ofrece mayor potencial para establecer una conexión entre ellas. Así lo refiere alguna literatura sobre Behavioral Law and Economics que ha tratado de apartarse de los modelos que marcan una distinción categórica entre racionalidad e irracionalidad y que, en lugar de ello, propone una comprensión más matizada de los procesos de toma de decisiones ${ }^{100}$. Esta literatura comienza por admitir que existirían brechas en la comprensión científica de tales procesos, toda vez que los mismos varían entre distintas culturas y entre individuos dentro de esas mismas culturas ${ }^{101}$. Según esta visión, las desviaciones de la asunción de racionalidad plena no son erráticas, sino que se presentan

94 Mitchell (2018) p. 459.

95 Jolls, Sunstein y Thaler (1998) p. 1474.

96 Pykett, Jones y Whitehead (2017) p. 7.

97 Mitchell (2018) p. 459.

98 Pykett, Jones y Whitehead (2017) p. 6.

99 Pykett, Jones y Whitehead (2017) p. 6.

100 Zamir y Teichman (2014) p. 151.

101 Mitchell (2018) p. 461; RachlinSKi (2008) pp. 870-875. 
en patrones predecibles y sistemáticos, pero que a su vez difieren en atención a factores individuales, sociales, demográficos, organizacionales y culturales ${ }^{102}$. En particular, puede ser útil tener en cuenta las denominadas teorías de las preferencias descubiertas, que solo admiten la validez de los axiomas de racionalidad respecto de quienes tienen incentivos adecuados y oportunidades efectivas para llegar a conocer y revelar sus verdaderas preferencias ${ }^{103}$.

Estas reflexiones plantean un serio cuestionamiento a la idea según la cual todas las personas son susceptibles por igual a la influencia del nudge y desafían las aproximaciones rígidas en torno a la aplicabilidad de las contribuciones de las ciencias conductuales al derecho ${ }^{104}$. Sin embargo, al mismo tiempo, ellas representan un margen para incorporar consideraciones relativas a la vulnerabilidad grupal en la teorización que subyace a estas regulaciones, con lo cual potencialmente podría incrementar su eficacia. Considerando este margen, las críticas al nudge fundadas en su potencial impacto negativo sobre grupos desaventajados no han necesariamente de tomarse como argumentos decisivos en contra de esta técnica. Antes bien, dichas críticas constituyen inquietudes legítimas que pueden y deben ser tomadas en cuenta.

Ahora bien, ¿qué aspectos en concreto de la teorización en torno al nudge ameritarían ser reinterpretados o modificados para incorporar el enfoque de la igualdad como no subordinación?

(a) En primer término, en línea con lo señalado más arriba, sería necesario abandonar concepciones rígidas acerca de la naturaleza humana y de la racionalidad humana. Esto obligaría a los arquitectos de decisiones a poner especial atención en la definición de los grupos destinatarios del nudge, en consideración a sus específicas características, entornos y necesidades. De igual manera, les exigiría considerar minuciosamente la evidencia empírica disponible en relación con la medida en que las observaciones de las ciencias conductuales en que se sostiene la intervención son generalizables a individuos que se encuentran en distintos contextos.

(b) Como segunda cuestión, la arquitectura de decisiones habría de redirigir sus esfuerzos y centrarse en promover la inclusión social y suprimir estereotipos y prácticas de subordinación, y no solo en incrementar el bienestar personal de los individuos "a juicio de ellos mismos". Ello, a su vez, implicaría aceptar que no todo nudge deba adscribirse a la filosofía del paternalismo libertario. De esta forma, podría morigerarse el componente individualista que suele asociarse a esta figura.

(c) Luego, en tercer lugar, sería preciso reconocer que existe una relación de complementariedad y refuerzo recíproco entre las distintas herramientas de regulación de las conductas sociales, a saber: mandatos, prohibiciones e incentivos. Este ajuste, una vez más, demandaría apartarse de la filosofía del paternalismo libertario y de su énfasis en una intervención estatal mínima. En vez, supondría admitir la necesidad de robustecer los efectos del nudge con otras aproximaciones regulatorias.

\footnotetext{
102 Zamir y Teichman (2014) p. 152.

103 Por todos, Mitchell (2018) p. 471.

104 Korobin (2011) pp. 1668-1673.
} 
Sin duda, todo lo anterior redundaría en un nudge con un rol bastante más modesto y menos que protagónico que el que promueven autores como Thaler y Sunstein. Pero, al mismo tiempo, se trataría de uno más sensible a las efectivas capacidades materiales de sus destinatarios para adherir o escapar a él y, por ello, acaso más eficaz. Sobre todo, sería uno cuyos eventuales beneficios podrían distribuirse de manera más igualitaria en la sociedad.

\section{CONCLUSIÓN}

1. En este trabajo se ha examinado la compatibilidad entre la teorización subyacente a la figura del nudge, por un lado, y los postulados en que se apoya la concepción de la igualdad como no subordinación, por el otro. El objetivo de este análisis era precisar si la primera admitía consideraciones relativas a la desigualdad estructural de sus destinatarios que pudieran orientar su diseño o, si por el contrario, excluía categóricamente tal posibilidad. Una de las principales motivaciones detrás de esta investigación radicó en la constatación de posibles efectos redistributivos desfavorables derivados de la aplicación de esta técnica, en desmedro de ciertos grupos sociales desaventajados.

2. Las ideas centrales a partir de las cuales se estructura el desarrollo teórico del nudge en Thaler y Sunstein dan cuenta de una determinada concepción de la persona, la sociedad y el Estado. Así se puede apreciar, sobre todo, en las nociones de racionalidad limitada y de arquitectura de decisiones, guiada por la filosofía del paternalismo libertario. De acuerdo con la primera, las personas son vistas como sujetos cuyos principales obstáculos a sortear en el libre desarrollo de sus proyectos de vida están dados por la configuración de los mecanismos modulares que dominan sus operaciones cognitivas. La segunda, por su parte, asume que el Estado puede y debe intervenir en la superación de tales obstáculos, pero que su intervención debe ser mínima y excepcional. De no ser así, se correría el riesgo de que la definición del bienestar individual quede enteramente en manos del Estado.

3. Por lo que respecta a la tesis de Fiss de la igualdad como no subordinación, por otro lado, ella refleja una comprensión de la sociedad como entidad estructurada en grupos. De acuerdo con esta lectura, los principales obstáculos que enfrentan los individuos en el libre desarrollo de sus proyectos de vida están dados por su adscripción a un determinado grupo social y a las dinámicas de dominación que se producen entre este y los demás. El derecho y las demás instituciones sociales tienden a reproducir esas dinámicas, haciéndolas más profundas y potencialmente perpetuándolas. De ahí que se justifique una intervención estatal robusta dirigida a erradicar los patrones de subordinación social, por medio de medidas de acción afirmativa que aspiren a mejorar el estatus social de los grupos desaventajados.

4. A partir de la comparación entre estas dos teorizaciones es posible concluir que -al menos en las respectivas versiones de Thaler y Sunstein, por una parte, y de Fiss, por la otra- ellas presentan discrepancias significativas en aspectos esenciales de su construcción. Desde este punto de vista, es muy difícil imaginar un nudge en cuyo diseño se puedan incorporar consideraciones de desventaja grupal y no subordinación. Pese a ello, en este trabajo se ha argumentado que ambas teorizaciones coinciden en asignar un rol clave al contexto en sus presupuestos descriptivos. Pero, si se toma en serio el contexto, no podría obviarse que los procesos de toma de decisiones no son determinados únicamente por fac- 
tores cognitivos, sino también por factores individuales y culturales. Esa observación, avalada por parte de la literatura sobre Behavioral Law and Economics, posibilitaría el diseño de nudges más eficaces y cuyos efectos sociales beneficiosos podrían distribuirse más igualitariamente en la sociedad.

\section{BIBLIOGRAFÍA}

AraYA, Sebastián y otros (2019): "Identifying Food Labeling Effects on Consumer Behavior". Disponible en: <https://ssrn.com/abstract=3195500> [consulta: 1 de junio de 2020].

BALDwin, Robert (2014): "From Regulation to Behaviour Change: Giving Nudge the Third Degree", The Modern Law Review, vol. 77, No 6: pp. 831-857.

Bovens, Luc (2009): “The Ethics of Nudge", en Grüne-Yanoff, Till y Hansson, Sven Ove (eds.), Preference Change: Approaches from Philosophy, Economics and Psychology (Dodrecht, Springer) pp. 207-219.

Breyer, Stephen (2005): Active Liberty: Interpreting Our Democratic Constitution (New York, Knopf).

Brownsword, Roger (2014): "Human Dignity, Human Rights, and Simply Trying to Do the Right Thing", en McCrudden, Christopher (ed.), Understanding Human Dignity (Oxford, Oxford University Press) pp. 345-358.

Button, Mark E. (2018): "Bounded Rationality without Bounded Democracy: Nudges, Democratic Citizenship. and Pathways for Building Civic Capacity", American Political Science Association, vol. 16, No 4: pp. 1034-1052.

Cantrell, Jennifer y otros (2013): "Impact of Tobacco-Related Health Warning Labels across Socioeconomic, Race and Ethnic Groups: Results from a Randomized WebBased Experiment", PLoS One, vol. 8, No 1: pp. 1-11.

Cassesse, Sabino (2016): "Exploring the Legitimacy of Nudging”, en Kemmerer, Alexandra y otros (eds.), Choice Architecture in Democracies: Exploring the Legitimacy of Nudging (Oxford, Hart Publishing) pp. 241-245.

Drichoutis, Andreas C., Lazaridis, Panagiotis y Nayga, Rodolfo M. (2005): "Nutrition knowledge and consumer use of nutritional food labels", European Review of Agricultural Economics, vol. 32, No 1: pp. 93-118.

Esparza Reyes, Estefanía (2017): La igualdad como no subordinación. Una propuesta de interpretación constitucional (Tirant lo Blanch, Ciudad de México).

Fiss, Owen M. (1971): "A Theory of Fair Employment Laws", The University of Chicago Law Review, vol. 38, No 2: pp. 235-314.

Fiss, Owen M. (1976): "Groups and the Equal Protection Clause", Philosophy \& Public Affairs, vol. 5, No 2: pp. 107-177.

Fiss, Owen M. (1984): "Against Settlement", The Yale Law Journal, vol. 93, No 6: pp. 1073-1090.

FISS, Owen M. (1986): "Free Speech and Social Structure", Iowa Law Review, vol. 71: pp. 1405-1425.

FIss, Owen M. (1987): “Why the State?", Harvard Law Review, vol. 100: pp. 781-794. 
FIss, Owen M. (1992): "Freedom and Feminism", The Georgetown Law Journal, vol. 80: pp. 2041-262.

Fiss, Owen M. (1994): "What is Feminism?”, Arizona State Law Journal, vol. 26: pp. 413-428.

Fiss, Owen M. (1996a): Liberalism Divided: Freedom of Speech and the Many Uses of State Power (Routledge, New York).

Fiss, Owen M. (1996b): The Irony of Free Speech (Cambridge, Harvard University Press).

FISs, Owen M. (2004): "Another Equality", Issues in Legal Scholarship: The Origins and Fate of Antisubordination Theory, vol. 2, No 1: Art. 20. Disponible en: <https://doi. org/10.2202/1539-8323.1051> [consulta: 1 de junio de 2020].

Gandy, Oscar y Nemorin, Selena (2018): “Toward a political economy of nudge: smart city variations", Information, Communication \& Society, vol. 22, No 14: pp. 2112-2126.

Goodwin, Morag (2016): "Architecture, Choice Architecture and Dignity", en Kemmerer, Alexandra y otros (eds.), Choice Architecture in Democracies: Exploring the Legitimacy of Nudging (Oxford, Hart Publishing) pp. 285-308.

Hansen, Pelle Guldborg y Jespersen, Andreas Maaløe (2013): "Nudge and the Manipulation of Choice: A Framework for the Responsible Use of the Nudge Approach to Behaviour Change in Public Policy", European Journal of Risk Regulation, vol. 4, No 1: pp. 3-28.

Haselton, Martie G., Nettle, Daniel, Andrews, Paul W. (2015): "The evolution of cognitive bias", en Buss, David M.(coord.), The Handbook of Evolutionary Psychology (New York, John Wiley \& Sons) pp. 968-987.

Hausman, Daniel M. y Welch, Brynn (2010): "Debate: To Nudge or Not to Nudge", The Journal of Political Philosophy, vol. 18, No 1: pp. 123-136.

Heffetz, Ori, O’Donoghue, Ted y Schneider, Henry S. (2020): "Reminders Work, But for Whom? Evidence from New York City Parking-Tickets Recipients". Disponible en: $<$ https://ssrn.com/abstract=2889749> [consulta: 1 de junio de 2020].

HorTon, Tim (2009): “The darker side of nudging”, The Political Quarterly, vol. 80, No 2: pp. 295-298.

HusaK, Douglas (2015): "Paternalism", en Marmor, Andrei (ed.), The Routledge Companion to Philosophy of Law (New York, Routledge) pp. 467-480.

JoHn, Peter y otros (2011): Nudge, nudge, think, think: Experimenting with ways to change civic behaviour (London, Bloomsbury Academic).

John, Peter; Smith, Graham; STOKer, Gerry (2009): "Nudge nudge, think think: two strategies for changing civic behaviour", The Political Quarterly, vol. 80, o 3: pp. 361-70.

John, Peter y STOKer, Gerry (2017): "From nudge to nudge plus: behavioural public policy for a self-guiding society", IPAA 2017 Research and Practice. Disponible en: $<$ http://www.governanceinstitute.edu.au/magma/media/upload/ckeditor/files/From\%20 nudge $\% 20$ to $\% 20$ nudge $\% 20$ plus $\% 20$ behavioural $\% 20$ public $\% 20$ policy $\% 20$ for $\% 20$ a\%20self-guiding\%20society.pdf> [consulta: 1 de junio de 2020].

Kahneman, David (2011): Thinking Fast and Slow (New York, Macmillan).

Karst, Kenneth L. (1977): "Foreword: Equal Citizenship Under the Fourteenth Amendment", Harvard Law Review, vol. 91, No 1: pp. 1-68. 
KIm, Sung-Yong, NaYga, Rodolfo M. y Capps, Oral (2001): "Food label use, self-selectivity, and diet quality", The Journal of Consumers Affairs, vol. 35, No 2: pp. 346-363.

Korobkin, Russell (2011): "What Comes after Victory for Behavioral Law and Economics”, University of Illinois Law Review, vol. 2011, No 5: pp. 1653-1674.

Loewenstein, George y Chater, Nick (2017): "Putting nudges in perspective", Behavioural Public Policy, vol. 1, No 1: pp. 26-53.

LORENC, Theo y otros (2012): "What types of interventions generate inequalities? Evidence from systematic reviews", Journal of Epidemiology and Community Health, vol. 67, No 2: pp. 190-193.

McCrudden, Christopher y King, Jeff (2016): “The Dark Side of Nudging: The Ethics, Political Economy, and Law of Libertarian Paternalism”, en Kemmerer, Alexandra y otros (eds.), Choice Architecture in Democracies: Exploring the Legitimacy of Nudging (Oxford, Hart Publishing) pp. 67-132.

Mitchell, Gergory (2018): "The price of abstraction", en Teitelbaum, Joshua C. y Zeiler, Kathryn (eds.), Research Handbook on Behavioral Law and Economics (Cheltenbaum, Elgar) pp. 459-475.

Morgan, Myfanwy y otros (2013): "Attitudes to deceased organ donation and registration as a donor among minority ethnic groups in North America and the UK: A syn-thesis of quantitative and qualitative research", Ethnicity \& Health, vol. 118, No 4: pp. 367-390.

National Advisory Committee on Bioethics (2015): A Report by the National Advisory Committee on Bioethics (Dublin, Department of Health).

Persad, Govind (2014): "Libertarian Patriarchalism: nudges, procedural roadblocks and reproductive choice”, Women's Rights Law Reporter, vol. 35, No 2: pp. 273-298.

Ponce de León Solís, Viviana (2019): "El nudge, su aplicación en el derecho chileno y sus potenciales problemas de constitucionalidad", Revista Chilena de Derecho, vol. 46, No 2: pp. 345-371.

Pykett, Jessica, Jones, Rhys y Whitehead, Mark (2017): "Introduction: psychological governance and public policy", en Pykett, Jessica, Jones, Rhys y Whitehead, Mark (eds.), Psychological Governance and Public Policy: Governing the Mind, Brain and Behaviour (Abingdon, Routledge) pp. 1-20.

Quigley, Muireann (2013): "Nudging for health: on public policy and designing choice architecture", Medical Law Review, vol. 21, No 4: pp. 588-621.

Rachlinski, Jeffrey (2008): "Heuristics, Biases, and Philosophy", Tulsa Law Review, vol. 43, No 4: pp. 865-883.

Roberts, Jessica L. (2018): "Nudge-Proof: Distributive Justice and the Ethics of Nudging", Michigan Law Review, vol. 116, No 6: pp. 1045-1066.

Simon, Herbert A. (1982): Models of Bounded Rationality. Volume 1: Economic Analysis and Public Policy (Cambridge, The MIT Press).

Jolls, Christine, Sunstein, Cass R. y Thaler, Richard (1998): "A Behavioral Approach to Law and Economics", Stanford Law Review, vol. 50: pp. 1471-1550.

Sunstein, Cass R. (2014): Why Nudge? The Politics of Libertarian Paternalism (New Haven, Yale University Press). 
Sunstein, Cass R. (2015): "The Ethics of Nudging", Yale Journal of Regulation, vol. 32, No 2: pp. 414-450.

Sunstein, Cass R. (2016a): "People Prefer System 2 Nudges (Kind of)”, Duke Law Journal, vol. 66, No 1: pp. 121-168.

Sunstein, Cass R. (2016b): The Ethics of Influence: Government in the Age of Behavioral Science (New York, Cambridge University Press).

Thaler, Richard H. y Sunstein, Cass R. (2008): Nudge: Improving Decisions about Health, Wealth, and Hapiness (New Haven, Yale University Press).

Thomas, Sian y otros (2008): "Population tobacco control interventions and their effects on social inequalities in smoking: systematic review", Tobacco Control, vol. 17: pp. 230-237.

Tversky, Amos y Kahneman, David (1974): "Judgment under Uncertainty: Heuristics and Biases”, Science, vol. 185, No 4157: pp. 1124-1131.

Van AaKen, Anne (2015): "Judge the Nudge: In Search of the Legal Limits of Paternalistic Nudging in the EU”, en Alemanno, Alberto y Sibony, Anne-Lise (eds.), Nudge and the Law, A European Perspective (Oxford, Bloomsbury) pp. 100-136.

Waldron, Jeremy (2014): "It's all for your own good", The New York Review of Books.

Wilkinson, T. M. (2013): "Nudging and Manipulation”, Political Studies, vol. 61: pp. 341-355.

Yeung, Karen (2011): "Can We Employ Design-Based Regulation While Avoiding Brave New World?”, Law, Innovation and Technology, vol 3, No 1: pp. 1-29.

Yeung, Karen (2012): "Nudge as Fudge", The Modern Law Review, vol. 75, No 1: pp. 122-148.

Young, Iris Marion (1990): Justice and the Politics of Difference (Princeton, Princeton University Press).

Zamir, Eyal y Teichman, Doron (2018): Behavioral Law and Economics (Oxford, Oxford University Press). 
